http://jmscr.igmpublication.org/home/ ISSN (e)-2347-176x ISSN (p) 2455-0450

crossref DOI: https://dx.doi.org/10.18535/jmscr/v8i2.46

\title{
Thrombotic Complications in Dilated Cardiomyopathy Patients- Comparative Study Using Echocardiography and Cardiac MRI
}

\author{
Authors \\ Dr Imtiyaz Ahmad Khan', Dr Manjeet Singh², Dr Rameez Mohiudin 3 \\ ${ }^{1}$ Fellow Thoracic Radiology, AIIMS \\ ${ }^{2}$ Professor, SKIMS Soura JK \\ ${ }^{3}$ Senior Resident, SKIMS Soura JK
}

\begin{abstract}
Objectives: Comparison between Echocardiography and Cardiac MRI in detection of intraventricular thrombi in Dilated cardiomyopathy patients.

Methods: Sixty Patients of DCM with reduced LV EF (<50\%) were enrolled in this study.

All echocardiographic studies were performed on Aloka USG by an experienced cardiologist.

All Cardiac MR Studies were performed on 1.5 Tesla MRI .Obtained data was analyzed on Siemens Workstation.

Data was analyzed using Analysis of variance technique (ANOVA), Pearson's correlational coefficient and Students $t$-test. A P-value of $<0.05$ was taken as significant. Bland-Altman method was used for comparison between EF values and ventricular volumes.

Observations: MRI showed ventricular thrombi in 9 out of 60 patients with average size of 1 to $9 \mathrm{~cm}^{3}$. Of the detected thrombi 6 were localized at ventricular apex and three were visualized at anteroseptal wall.

Trans-thoracic Echocardiography showed ventricular thrombus in 2 patients located in LV apex with average size of $4 \mathrm{~cm} 3$.

Taking MRI as gold standard for detection of intra-ventricular thrombi; Sensitivity of Echo For Thrombi Detection $=33.4 \%$.

Difference in EF on MRI between patients with and without ventricular thrombi was $8.96(P=0.020)$.

Presence of intraventricular thrombi correlated with an increased left ventricular end diastolic volume. $(P=0.010)$

Conclusion

1) CARDIAC MRI is a better modality in identification of LV thrombi in DCM patients.

2) Presence of intra-ventricular thrombi correlates with lower EF and increased Left ventricular end diastolic volumes.
\end{abstract}

\section{Introduction}

Dilated cardiomyopathy (DCM), the most common cardiomyopathy Worldwide with a prevalence of $40-50$ cases per $100,000^{(1)}$, is characterized by an increase in diameter and volume of the left or both ventricles (globular Left
Ventricle with LVIDD -Left ventricular internal diastolic diameter $>56 \mathrm{~mm}$, LV sphericity index ratio < 1.5) and leading to impaired systolic Ejection fraction $(\mathrm{EF}<40 \%)$, that is not secondary to or cannot be exclusively justified by abnormal loading conditions (e.g. valve disease, 
hypertension) or by the concomitant coronary artery disease (CAD). ${ }^{(2)}$

Incidence of L.V thrombi is estimated to be $13 \%$ in DCM patients with sinus rhythm and depressed $\mathrm{EF}(<35 \%) .{ }^{(3)} \mathrm{LV}$ followed by $\mathrm{RV}$ is the most common site of thrombi formation in $\mathrm{DCM}^{(3)}$. Apex is the most common location in left ventricular thrombi formation ${ }^{(4)}$. Among DCM patients with LV Thrombi concomitant associated $\mathrm{RV}$ (Right ventricle) thrombus is reported in $15 \%$ of patients ${ }^{(4)}$.

Recognition and appropriate treatment of thrombotic complication in DCM is necessary to prevent systemic thromboembolism which is estimated to occur in $4 \%$ of patients with DCM who have Ventricular EF $<35 \%$. $^{(5,6,7)}$

Causation of Thrombo-embolism in DCM is a multifactorial process with variable interplay between various individual factors like impaired LV function, stasis of blood flow, dysrhythmias, hypercoagulable state and abnormal procoagulant ventricular surface in $\mathrm{DCM}^{(8)}$.

In clinical practice LV thrombi are diagnosed by transthoracic echocardiography (TTE) ${ }^{(9)}$ which however is limited by less overall accuracy. Transesophageal echocardiography (TEE) has improved sensitivity and specificity however limited by its invasive nature and requirement of anesthesia. Recent

Studies with contrast- enhanced (CE)-MRI have shown promising results in diagnosing ventricular thrombi with improved sensitivity and specificity and Overall accuracy. ${ }^{(10,11,12)}$

\section{Methods}

Sixty patients of DCM with reduced LV EF $(<50 \%)$ were prospectively recruited in this study. M-Mode Echocardiography was performed in all patients however only 42 (70\%) patients had adequate window to undergo 2D Echocardiography. All patients underwent Cardiac MRI within two weeks of the preliminary echocardiography. All studies were performed according to the guidelines of the hospital committee on medical ethics and clinical investigation. Informed consent was obtained from all patients.

Patients with already established coronary artery disease (CAD), LV dysfunction due to a specific cause like drugs, CKD-Chronic Kidney Disease. Stage $3 \& 4$ and with history of contrast hypersensitivity were not enrolled.

\section{Examination Techniques and Imaging Protocols}

\section{Echocardiography}

All echocardiographic studies were performed on Aloka USG System (Model IPF-1504; SN M03761).M-mode and 2D-Echocardiography were performed by two experienced Cardiologists. A standardized protocol was performed with cross-sectional imaging of left-ventricle immediately distal to mitral valve tips and apical two-dimensional imaging based on orthogonal 2 and 4-chamber views.

\section{Cardiac MRI}

All MR (Magnetic Resonance) studies were performed on 1.5 Tesla MR System (Magnetom Avanto, Siemens Medical System). After the preliminary localizing

Sequence, the imaging protocol included:

Turbo spin echo (tse) sequences for morphology.

SSFP \& FLASH Sequences for ventricular function.

Segmented T1W TIRM sequences for delayed enhancement imaging.

Post contrast T1-weighted turbo spin-echo imaging, after injection of $0.1 \mathrm{mmol} / \mathrm{kg}$ of Gadolinium-diamide (Omniscan), using a powerinjector followed by $20 \mathrm{ml}$ saline flush.

The sequences were taken in axial/sagittal/ coronal/oblique planes as required.

The basal short axis view was taken just ahead of atrioventricular ring and subsequent cines were taken in $1 \mathrm{~cm}$ steps towards LV apex.

An average of 10 short axes segments were taken to encompass the entire LV.

Obtained data was then posted on PACS and analyzed on a Siemens MR Workstation. Enddiastolic and end-systolic images were chosen as 
images having maximum and minimum left ventricular diameters respectively in a Cinematic display .Short axis end-diastolic and end-systolic endocardial borders were traced manually for each slice, then multiplied by slice thickness and finally added up to obtain EDV and ESV. Ejection fraction was then calculated as EDV-ESV/EDV. Papillary muscles were excluded from the measurement and care was taken not to include left atrium in volumetry in end systolic phase.

\section{Results}

\section{Detection of Intraventricular Thrombi}

Ventricular thrombi were detected in 9 patients (15\%) on Cardiac MRI with average size of 1 to 9 $\mathrm{cm}^{3}$. Of the detected thrombi 6 were localized at ventricular apex and three were visualized at anteroseptal wall. On MRI intra ventricular thrombus followed myocardial signal intensity on all sequences and appeared as non-enhancing filling defect within the ventricular cavity on post contrast MRI. All the nine thrombi were equally visualized in cine MRI and post-contrast MRI.

Trans-Thoracic Echocardiography showed ventricular thrombus in 2 patients with average size of $4 \mathrm{~cm}^{3}$ and both the ventricular thrombi were visualized in LV apex.

All the ventricular thrombi visualized on cardiac MRI but not on Echo ranged in size from $1-3 \mathrm{~cm}^{3}$.

\section{Comparison of Thrombi Detection between Echo and MRI}

\begin{tabular}{|c|c|c|c|c|}
\hline \multirow{2}{*}{} & \multirow{3}{*}{ MRI } & $\mathrm{A}$ & $\mathrm{P}$ & \multirow{2}{*}{ Total } \\
\cline { 3 - 5 } & \multirow{2}{*}{$\mathrm{A}$} & $\begin{array}{c}51 \\
(91.9 \%)\end{array}$ & 0 & 34 \\
& \multirow{2}{*}{$\mathrm{P}$} & $\begin{array}{c}7 \\
(8.1 \%)\end{array}$ & $\begin{array}{c}2 \\
(100.0 \%)\end{array}$ & $\begin{array}{c}6 \\
(15.0 \%)\end{array}$ \\
\hline Total & & 38 & 2 & 40 \\
\hline
\end{tabular}

\section{THROMBUS DETECTION}

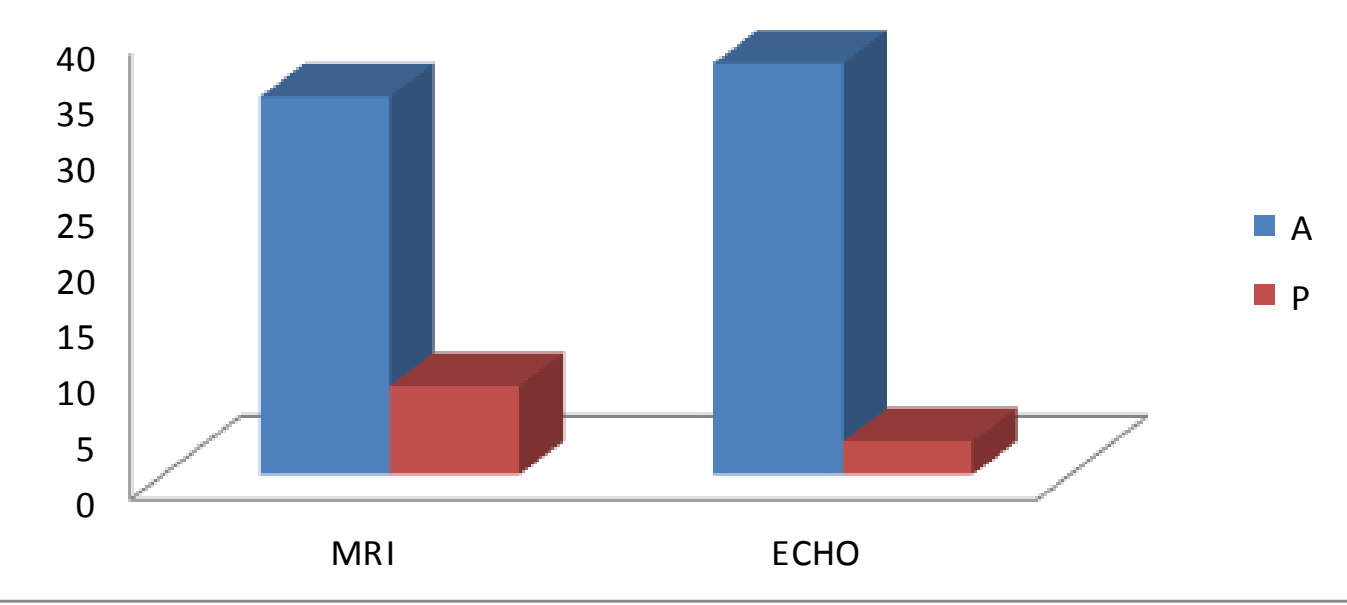

Taking MRI as gold standard for detection of intra-ventricular thrombi; Sensitivity of Echo (TTE) For Thrombi Detection is $33.4 \%$.

On comparing the two groups of patients (with and without thrombi) with respect to their ejection fraction as determined by MRI, the mean EF of patients with thrombi was $23.0 \%$ as compared to the mean $\mathrm{EF}=31.96 \%$ of patients without thrombi. The mean difference between the two groups was 8.96 which is statically Significant $(\mathrm{P}=0.020)$ as shown in table 2 . 
Table 2: Comparison of Ejection Fraction between Patients with and Without Thrombi

\begin{tabular}{|l|c|c|}
\hline & \multicolumn{2}{|c|}{ Ejection fraction } \\
\hline & Without Thrombi & With Thrombi \\
\hline Number of patients(n) & 34 & 6 \\
\hline Mean EF & 31.96 & 23.0 \\
\hline Std. Deviation & \multicolumn{2}{|c|}{8.96154} \\
\hline Mean Difference & \multicolumn{2}{|c|}{0.020} \\
\hline P-value & \multicolumn{2}{|c|}{1.487 to 16.436} \\
\hline $\begin{array}{l}\text { 95\% Confidence Interval of } \\
\text { the Difference }\end{array}$ &
\end{tabular}

Similarly presence of intraventricular thrombi correlated with an increased LVEDV (left ventricular end diastolic volume).In patients with thrombimean LVEDV was 219+/-67mlas compared to $\mathrm{LVEDV}=180+/-60 \mathrm{ml}$ in patients without thrombi which is spastically significant $(\mathrm{P}=0.010)$.

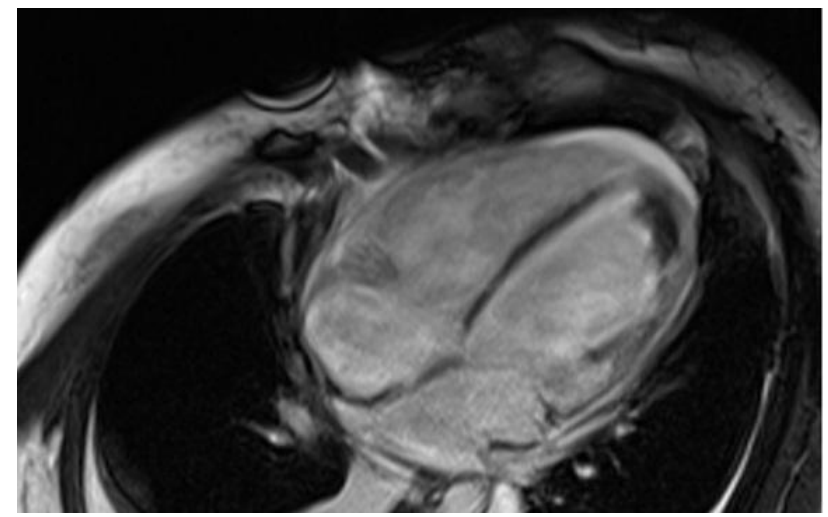

Fig 01

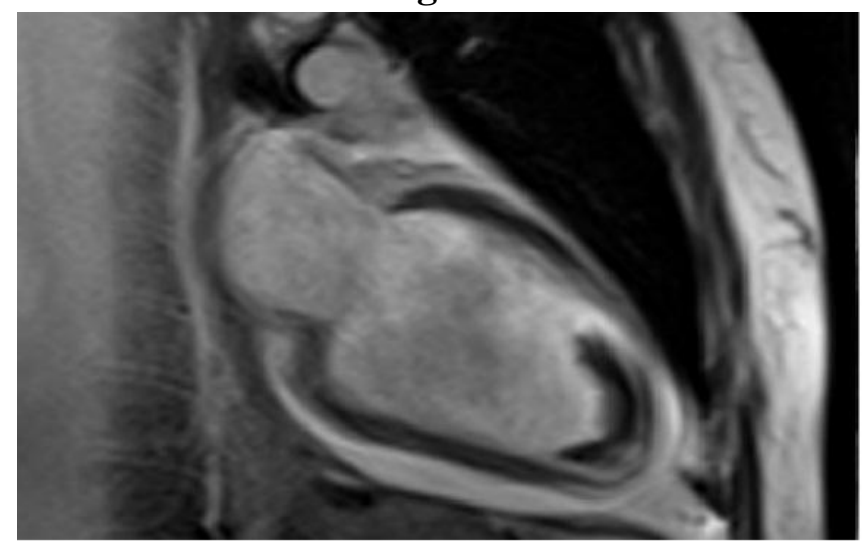

Fig 01

\section{Discussion}

Ventricular thrombi formation in DCM patients is not an uncommon occurrence with an incidence of $15 \%$ in our study population. CEMRI is a much better investigation in diagnosis of ventricular thrombi in DCM as compared to TTE with comparative Sensitivity of Echo (TTE) For Thrombi Detection being 33.4\% only, in our study. Non--visualized thrombi on TTE are much smaller with mean volume being less than $4 \mathrm{~mm}^{3}$.

Non-contrast Echo detects thrombus based on anatomical characteristics thus having high sensitivity in large and protuberant thrombi but limited accuracy in small and mural thrombi. Poor acoustic window due to physical attributes of patient is one of the factors for decreased detection of ventricular thrombi in Echocardiography.

In comparison, CEMRI uses both anatomical and tissue characterization approaches for detection of ventricular thrombi. Due to homogenous opacification of ventricular cavity after gadolinium administration, abnormal intraventricular structures will have a dark appearance. This allows the visualization of small thrombi invisible on TTE.

We compared patients with and without thrombus formation on CE-MRI. The mean $\mathrm{EF}$ of patients with thrombi was significantly lower as compared to the mean EF of patients without thrombi. The mean difference between the two groups is statically Significant $(\mathrm{P}=0.020)$. Similar statically significant difference was found in LVEDV in the two groups of patients.

Increased LVEDV and depressed EF might contribute to increased risk of thrombi formation by altering the pump hemodynamics resulting in increased stasis of blood with variable contribution from other factors like

Increased coagulability and thrombogenic ventricular endothelium. 
These results are consistent with literature. NICO.R.MOLLET et al in $2002^{(13)}$ assessed visualization of intra-ventricular thrombus with contrast-enhanced MRI in patients with ischemic heart disease, studied 57 patients of IHD with CEMRI and found intra-ventricular thrombi in $21 \%$ $(n=12)$ of patients whereas ECHO could pick thrombus in only $8.78 \%(\mathrm{n}=5)$ and demonstrated sensitivity of $\mathrm{ECHO}=41.6 \%$ in picking thrombus (compared to $33.4 \%$ in our study). The subgroup of patients with intra-ventricular thrombus correlated with larger EDV, lower EF, lower wall motion score and larger areas of delayed enhancement.

Bruder $\mathrm{O}$ et al in $2005^{(14)}$ in a study to evaluate detection and characterization of left ventricular thrombi by MRI compared to transthoracic echocardiography studied 82 patients (55 men and 27 women, age 36 to 79 years, median $59+/-11$ years) who suffered a myocardial infarction more than 6 months earlier, with transthoracic echocardiography and MRI. Transthoracic echocardiography depicted 12 thrombi. Contrastenhanced MRI confirmed these 12 thrombi and detected 23 additional thrombi concluding that Contrast-enhanced MRI is mostly superior to transthoracic echocardiography in diagnosing mural left ventricular thrombi in patients who had suffered a myocardial infarction.

Intracavitary thrombi are more frequently found in patients with impaired regional and global left ventricular function

\section{Conclusion}

1) Cardiac MRI is a better modality in identification of LV thrombi in DCM patients surpassing TTE.

2) Presence of intra-ventricular thrombi on Cardiac MRI correlates with lower EF and increased Left ventricular end diastolic volumes.

\section{Bibliography}

1. Roger VL, Go AS, Lloyd-Jones DM, et al. Heart disease and stroke statistics - 2011 update: a report from the American Heart Association Circulation 2011 ; 123 (4): e18-e209.

2. W. F. Armstrong and T. Ryan, Dilated Cardiomyopathy.

Feigenbaum's

Echocardiography, Lippincott Wilkins \& Wilkins, Philadelphia, $\mathrm{Pa}, \mathrm{USA}, 7^{\text {th }}$ edition, 2010

3. J. S. Gottdiener, J. A. Gay, L. Van Voorhees, R. DiBianco, and R. D. Fletcher, "Frequency and embolic potential of left ventricular thrombus in dilated cardiomyopathy: assessment by 2 dimensional echocardiography," The American Journal of Cardiology, vol. 52, no. 10, pp. 1281-1285, 1983.

4. M. Billingsley and H. Leong-poi, "Left ventricular thrombus: diagnosis, prevention and management," Cardiology Rounds, vol. X, no. 7, 2005

5. N. D. Sharma, P. A. McCullough, E. F. Philbin, and W. D. Weaver, "Left ventricular thrombus and subsequent thromboembolism in patients with severe systolic dysfunction," Chest, vol. 117, no. 2, pp. 314-320, 2000.

6. Nair, B. Sealove, J. L. Halperin, G. Webber, and V. Fuster, "Anticoagulation in patients with heart failure: who, when, and why?" European Heart Journal, Supplement, vol. 8, pp. E32-E38, 2006

7. Vaitkus PT, Barnathan ES. Embolic potential, prevention and management of mural thrombus complicating anterior myocardial infarction: a meta-analysis. J Am Coll Cardiol. 1993;22:1004-1009.

8. R. Delewi, F. Zijlstra, and J. J. Piek, "Left ventricular thrombus formation after acute myocardial infarction," Heart, vol. 98, no. 23, pp. 1743-1749, 2012.

9. Visser CA, Kan G, David GX, et al. Twodimensional echocardiography in the diagnosis of left ventricular thrombus: a prospective study of 67 patients with 
anatomic validation. Chest. 1983;83:228232.

10. Bruder O, Waltering KU, et al. Detection and characterization of left ventricular thrombi by MRI compared to transthoracic echocardiography. Rofo. 2005 Mar; 177 (3):344-9.

11. Lipke C, Katoh $\mathrm{M}$ et al. The value of noncontrast harmonic transthoracic echocardiography for the detection of left ventricular thrombi in patients with cardiomyopathy: comparison with contrast-enhanced magnetic resonance imaging. Int J Cardiovasc Imaging. 2007 Aug; 23(4): 479-87

12. J. W. Weinsaft, H. W. Kim, A. L. Crowley et al., "LV thrombus detection by routine echocardiography: insights into performance characteristics using delayed enhancement CMR," Journal of the American College of Cardiology, vol. 4, no. 7, pp. 702-712, 2011.

13. Nico R. Mollet, Steven Dymarkowsk et al. Visualization of Ventricular Thrombi With Contrast-Enhanced Magnetic Resonance Imaging in Patients With Ischemic Heart Disease. Circulation 2002;106:2873-2876.

14. Bruder O, Waltering KU, et al. Detection and characterization of left ventricular thrombi by MRI compared to transthoracic echocardiography. Rofo. 2005 Mar;177 (3):344-9. 